\title{
Technè
}

La science au service de l'histoire de l'art et de la préservation des biens culturels

45 | 2017

Bronzes grecs et romains : études récentes sur la statuaire antique

\section{Les bronzes noirs antiques - nouvelles observations et mécanismes de création}

Antique black bronzes; new observations and creative techniques

Marc Aucouturier, François Mathis et Dominique Robcis

\section{OpenEdition}

1 Journals

Édition électronique

URL : http://journals.openedition.org/techne/1345

DOI : 10.4000/techne.1345

ISSN : 2534-5168

Éditeur

C2RMF

Édition imprimée

Date de publication : 1 mai 2017

Pagination : 114-123

ISBN : 978-2-7118-6408-9

ISSN : 1254-7867

Référence électronique

Marc Aucouturier, François Mathis et Dominique Robcis, «Les bronzes noirs antiques - nouvelles observations et mécanismes de création », Technè [En ligne], 45 | 2017, mis en ligne le 19 décembre 2019, consulté le 24 juillet 2020. URL : http://journals.openedition.org/techne/1345 ; DOI : https:// doi.org/10.4000/techne.1345

\section{cc) (1)}

La revue Technè. La science au service de l'histoire de l'art et de la préservation des biens culturels est mise à disposition selon les termes de la Licence Creative Commons Attribution - Pas d'Utilisation Commerciale - Pas de Modification 4.0 International. 


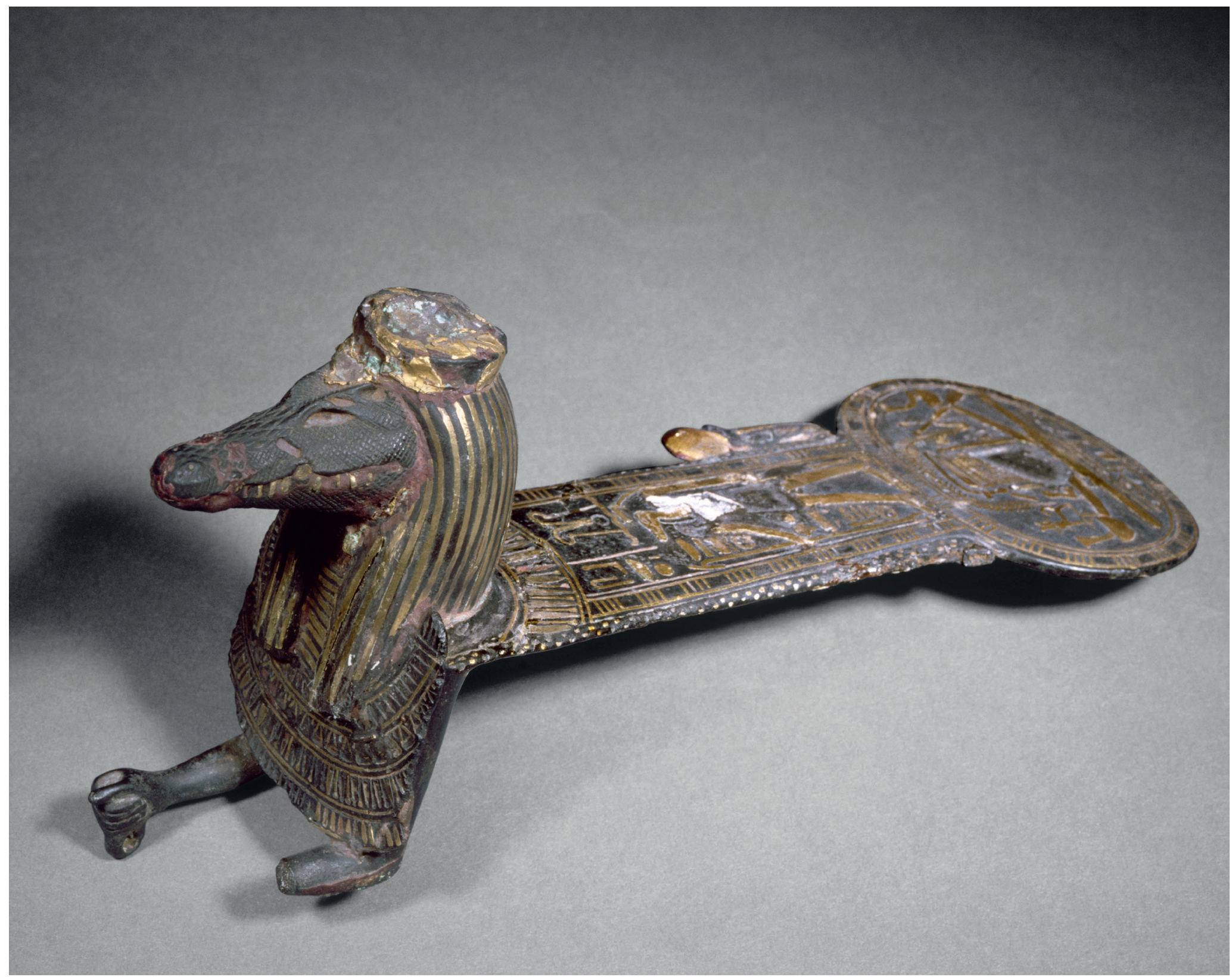

Fig. 1. Objet égyptien en bronze noir : contrepoids de collier Sobek (Nouvel Empire), musée du Louvre, département des Antiquités égyptiennes, inv. E 11520. L. 18,75 cm. ○ RMN-Grand Palais (musée du Louvre)/Hervé Lewandowski. 
Marc Aucouturier

François Mathis

Dominique Robcis

\section{Les bronzes noirs antiques - nouvelles observations et mécanismes de création}

Antique black bronzes; new observations and creative techniques
Résumé. Les bronzes noirs sont connus depuis le Moyen Empire égyptien; leur existence est ensuite attestée à Mycènes, en Grèce et dans la Rome antique, puis au Japon à l'époque moderne. Il s'agit d'une technique de patinage volontaire des alliages du cuivre par des procédés chimiques dont seules les recettes japonaises sont connues. Après une courte introduction historique et quelques considérations sur la structure et la composition des patines de bronzes noirs, cet article fait état d'observations et de reconstitutions de laboratoire inédites. Il s'attache à proposer des interprétations électrochimique et physique sur les mécanismes de formation et les propriétés optiques des couches de patine noire caractéristiques des objets obtenus par ces procédés.

Mots-clés. Patine, bronze noir, Égypte, Grèce, Rome, Japon, électrochimie, analyses par faisceaux d'ions, microscopie électronique en transmission.
Abstract. Black bronzes have been known to exist since the Middle Kingdom of Egypt: proof of their presence has been found in Mycenae, in Greece and in Ancient Rome, and, in the modern period, in Japan. The black surface was deliberately produced through a patination technique in which copper alloys were chemically treated. Only the Japanese formulas are known today. After a brief historical introduction and a few thoughts on the structure and composition of the black bronze patinas, the paper presents hitherto-unpublished observations and laboratory reconstructions. It seeks to propose physical and electrochemical interpretations of how the black patina was formed and the optical properties of the layered surfaces characteristic of the objects obtained through these processes.

Keywords. Patina, black bronze, Egyptian Antiquity, Roman Antiquity, electrochemistry, ion beam analysis, transmission electron microscopy.

\section{Introduction}

Le patinage intentionnel ${ }^{1}$ des bronzes, qui permet, grâce à un traitement chimique d'attaque de la surface, de modifier leur couleur, était pratiqué par les artisans bronziers dès l'Antiquitée . Cette technique a donné naissance à une qualité d'alliages appelée génériquement bronze noir. Connue en Égypte dès le Moyen Empire ${ }^{3}$, elle est également attestée dans le monde égéen et à l'époque romaine ${ }^{4}$. Sa pratique est aussi mentionnée en Chine, en Inde et surtout dans le Japon moderne (du XIV ${ }^{\mathrm{e}}$ siècle à nos jours) où elle est connue sous le nom de procédé nikomi shakushoku appliqué aux alliages $s h a k u d o^{5}$. Si les procédés utilisés au Japon (recettes de compositions des solutions, températures et temps de traitement) sont bien répertoriés ${ }^{6}$, les procédés de l'Antiquité sont en revanche très peu décrits et les documents existants ne sont pas suffisamment clairs pour être facilement interprétés. La première partie de cet article tentera de faire un point très résumé sur cet aspect historique. La définition scientifique du bronze noir ou du cuivre noir sera rappelée dans une deuxième partie, où seront brièvement résumées les descriptions données d'objets antiques en bronze intentionnellement patinés en noir. Nous tenterons également de comprendre comment la patine de cuprite $\mathrm{Cu}_{2} \mathrm{O}$ (de couleur naturelle rouge) adopte une couleur noire ou bleu-noir grâce au choix des alliages. Puis sera relatée une nouvelle étude de bronze noir romain mis en évidence sur un paludamentum faisant partie des trouvailles de Volubilis (Maroc). Nous engagerons enfin une discussion fondée sur des résultats expérimentaux récents, sur les mécanismes chimiques et électrochimiques mis en jeu dans l'obtention de cette patine particulière, ainsi que sur les mécanismes physiques qui créent sa couleur si recherchée par les artisans et les artistes. Le présent article se limite aux époques antiques, sauf en ce qui concerne les reconstitutions en laboratoire. Il ne prend pas en compte l'élaboration de patines par les procédés modernes apparus à la Renaissance, souvent orientés vers l'emploi de revêtements organiques, sans que les procédés fondés sur une attaque chimique du métal aient complètement disparu ${ }^{7}$.

Marc Aucouturier, ancien directeur de recherche émérite au CNRS, ancien membre du C2RMF, CNRS UMR 171 (marc.aucouturier@wanadoo. fr). François Mathis, chercheur archéomètre, Recherche et Prospection Archéologique (association sans but lucratif) (francois.mathis@ gmail. com). Dominique Robcis, chef de travaux d'art, C2RMF (dominique.robcis@culture.gouv.fr). 


\section{Considérations historiques, procédés connus et inconnus}

Les origines du perfectionnement de la polychromie métallique au moyen de patines intentionnelles sont imprécises. Une publication récente ${ }^{8}$, émanant de spécialistes, fait l'analyse de textes anciens, rédigés par des alchimistes grecs, dans lesquels on peut trouver de nombreuses informations techniques sur ces procédés. Les travaux de Marcellin Berthelot ${ }^{9}$ et l'examen des Papyri de Leyde et de Stockholm, repris et complétés par Robert Halleux, sont des sources intéressantes ${ }^{10}$. Ces documents présentent de nombreuses recettes (compositions de bains d'attaque) et procédés de traitement des métaux, souvent pour les faire ressembler à l'or, mais la seule information à caractère technique sur l'obtention des bronzes noirs aurait été trouvée dans une transcription au $\mathrm{XV}^{\mathrm{e}}$ siècle de la version syriaque d'un manuscrit (incomplet) de Zosime, l'un des alchimistes grecs (III ${ }^{\mathrm{e}}$ siècle) ; la traduction a été discutée par des auteurs anglophones en $2001^{11}$. La patine noire de ces bronzes est toujours obtenue par voie chimique, éventuellement accompagnée d'une étape thermique.

La plus ancienne œuvre connue de bronze patiné en noir est un petit crocodile conservé à Munich (fig. 1), daté du

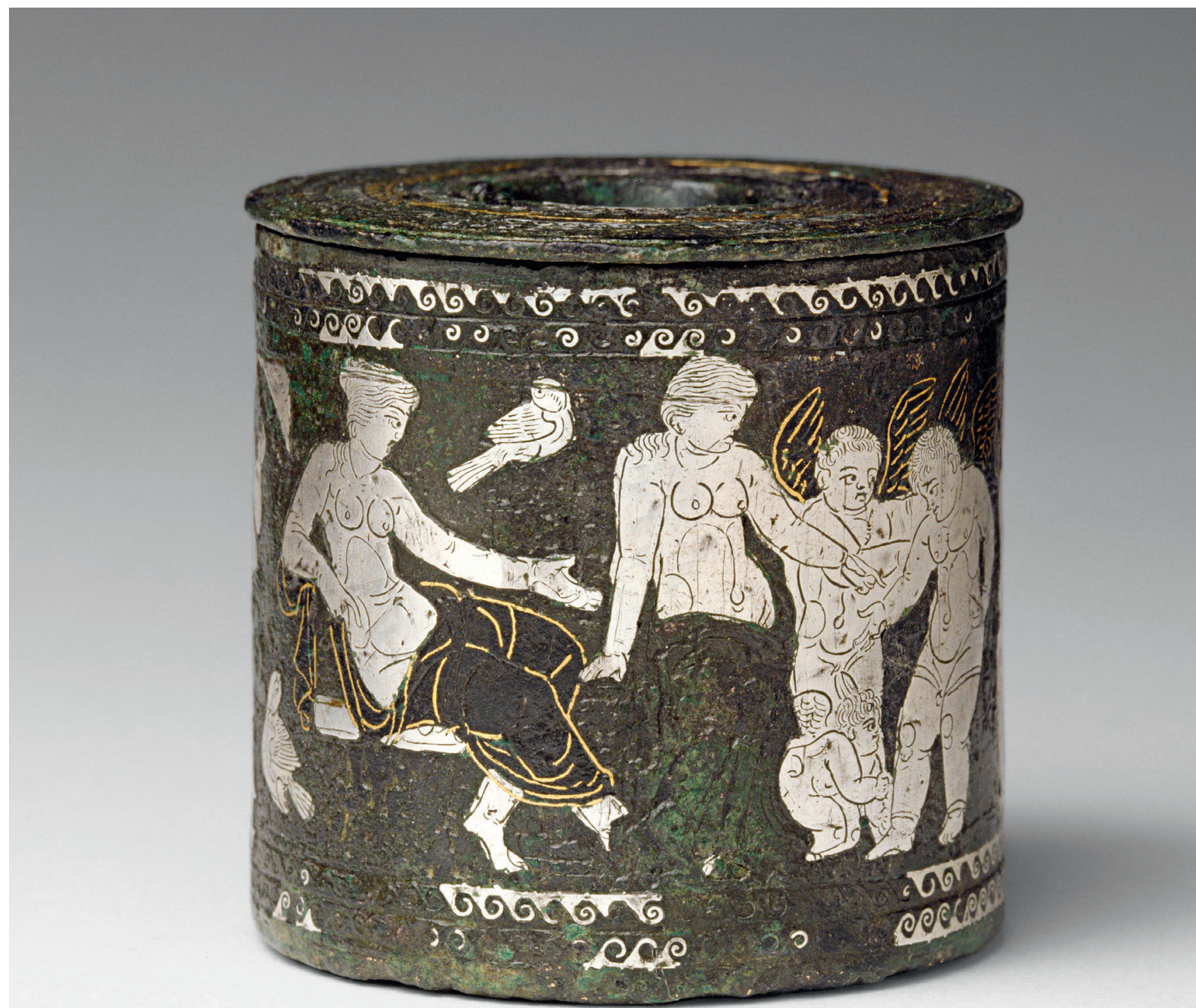

Fig. 2. Objet romain orné d'incrustations en bronze de Corinthe : encrier

de Vaison-la-Romaine ( ${ }^{\mathrm{er}}$ siècle), musée du Louvre, département des AGER,

inv. Bj 1950, H. 4,4 cm. (c) RMN-Grand Palais (musée du Louvre)/Hervé Lewandowski. 
Moyen Empire égyptien (env. 2003-1786 avant notre ère) ${ }^{12}$. Les bronziers égyptiens ont ensuite produit beaucoup d'objets pour la plupart cultuels, dont un certain nombre est conservé au musée du Louvre ${ }^{13}$ et au British Museum ${ }^{14}$. Leurs dates de production s'échelonnent du Moyen Empire à l'époque Ptolémaïque. Signalons que la mention égyptienne Hsmn-Km retraduite en $H m t y-K m$, hiéroglyphe signifiant respectivement bronze noir et cuivre noir, apparaît dans les inscriptions dès la XVIII ${ }^{\mathrm{e}}$ Dynastie (1550-1292 avant notre ère) $)^{15}$.

Le Sobek de la figure 1 constitue un exemple parmi la dizaine d'objets du département des Antiquités égyptiennes étudiés au C2RMF ${ }^{16}$.

La fabrication des bronzes noirs apparaît ensuite à Mycènes où ont été découvertes des dagues célèbres, incrustées de bronze noir, lui-même réincrusté de riches décors en or et argent. Ces dagues ${ }^{17}$, de fabrication probablement minoenne, datent du XVI ${ }^{\mathrm{e}}$ siècle avant notre ère.

En ce qui concerne la période grecque, l'existence d'objets pouvant être couverts de patine noire est rapportée par les textes ${ }^{18}$. On pense que le terme kyanos - traduit classiquement par bleu ou bleu noir - peut, lorsqu'il est utilisé pour un objet métallique, se rapporter à cette technique. Ainsi même un texte poétique comme la description du bouclier d'Achille par Homère devient compréhensible et plausible ${ }^{19}$. Malheureusement, à notre connaissance, il n'existe actuellement plus d'objets patinés datant de ces périodes qui soient susceptibles d'être analysés.

Pendant la période romaine, les bronzes noirs prennent, pense-t-on, le nom de bronzes de Corinthe, très prisés des élites. Il existe vraisemblablement beaucoup d'objets ou d'œuvres d'art décorés ou fabriqués avec cet alliage fameux à l'époque, cité par Pline l'Ancien dans son Histoire Naturelle ${ }^{20}$, ainsi que par plusieurs auteurs latins ${ }^{21}$. Parmi ces objets, l'un des plus anciens et des mieux connus, grâce à son étude approfondie au C2RMF, est l'encrier de Vaison-la-Romaine (fig. 2), conservé au musée du Louvre, département des AGER (inv. Bj 1950), daté avec précision du troisième quart du $\mathrm{I}^{\mathrm{er}}$ siècle de notre ère ${ }^{22}$.

Concernant les recettes permettant d'obtenir chimiquement la patine noire, seules sont connues celles utilisées pour les objets japonais en alliage shakudo, mais rien ne prouve qu'elles aient une parenté quelconque avec les recettes antiques. Faute d'informations précises sur les procédés et recettes antiques, et compte tenu des similitudes de compositions des alliages spécifiquement choisis par les artisans, similaires quelles que soient les périodes, ce sont ces recettes modernes qui sont employées par les chercheurs pour recréer des bronzes noirs dans le but de comprendre les mécanismes d'élaboration de la patine noire et ses propriétés physiques, comme nous le verrons plus loin.

\section{Rappels sur la structure et la composition des patines noires}

La thèse de François Mathis ${ }^{23}$ et quelques publications associées ${ }^{24}$ résument bien la majorité des données scientifiques qui caractérisent les bronzes noirs antiques, ainsi que les moyens d'étude permettant d'acquérir ces données sur des objets de musées : les bronzes noirs sont obtenus à partir d'alliages de cuivre ou bien de cuivre non allié auxquels ont été ajoutées de petites quantités d'or et/ou d'argent. Les teneurs de ce ou ces éléments nécessaires sont faibles et ne dépassent jamais quelques pourcents. Les études permettent de donner une définition précise d'un bronze noir ou d'un cuivre noir : un bronze noir ou un cuivre noir est un alliage de cuivre ou un cuivre non allié comportant de faibles additions d'or et/ou d'argent, dont la surface a été intentionnellement traitée par voie chimique pour provoquer la formation d'une couche de cuprite $\left(\mathrm{Cu}_{2} \mathrm{O}\right)$ de couleur noire ou bleu foncé, couche qui contient des quantités non négligeables d'or et/ou d'argent. Ce qui caractérise la patine des bronzes noirs est donc la présence de cuprite de couleur noire, différente de la couleur rouge présentée par ce composé à l'état naturel.

À partir d'une telle définition, il est possible d'identifier sans risque d'erreur cette catégorie de patine à dominante noire grâce à une simple analyse élémentaire non-destructive (par fluorescence X par exemple), doublée obligatoirement d'une identification de la structure de la couche de patine, par exemple par diffraction de rayons X ou par spectrométrie Raman. Cette précision de définition et cette rigueur dans les moyens analytiques sont nécessaires pour tenter de décrire et de comprendre un usage de la patine chimique dans le monde antique. En effet, nos travaux ont mis en évidence, outre les bronzes noirs, des patines chimiques noires, au soufre, sur des alliages argent-cuivre (plaque à l'aurige, musée du Louvre, département des AGER, inv. Br 3447) ${ }^{25}$, une patine chimique rouge sur laiton (strigile romain, musée du Louvre, département des AGER, inv. Br 1582) ${ }^{26}$, et d'autres patines noires sur alliages cuivreux possiblement intentionnelles, obtenues par voie thermique et non chimique.

\section{Observations récentes}

\section{Le paludamentum de Volubilis}

Parmi les chefs-d'œuvre présentés au MUCEM lors de l'exposition Splendeurs de Volubilis; Bronzes antiques du Maroc et de Méditerranée à Marseille, en mars 2014, figure un fragment de paludamentum (manteau de général ou d'empereur) en bronze ayant appartenu à une statue impériale, représentant peutêtre l'empereur Caracalla (188-217). Cet objet exceptionnel, trouvé au cours des fouilles de Volubilis, est conservé au musée archéologique de Rabat $^{27}$ (fig. 3). Il a été analysé par les auteurs du présent article dans les locaux du MUCEM quelques jours avant l'exposition ${ }^{28}$. Il comporte un décor très riche 


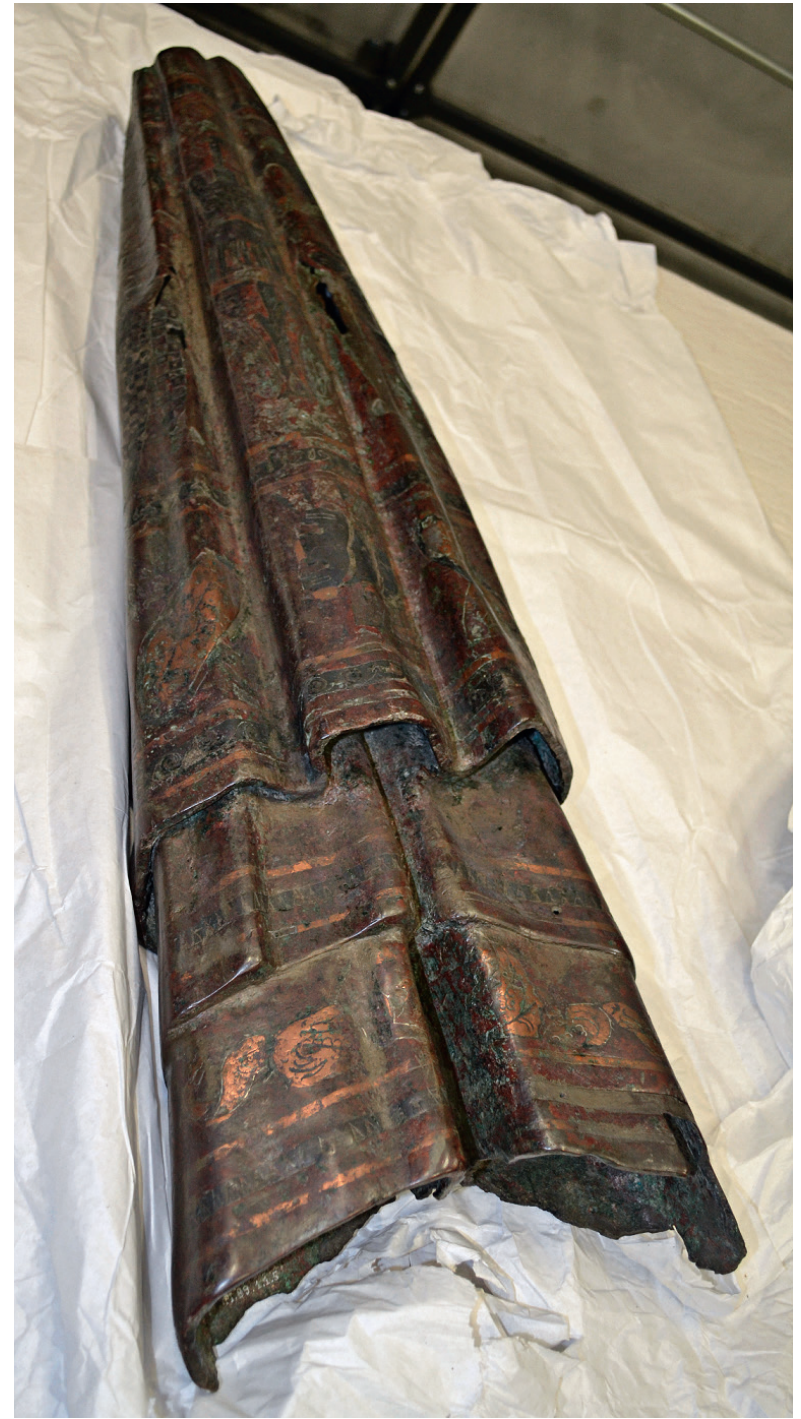

Fig. 3. Paludamentum en bronze de Volubilis, musée archéologique de Rabat (inv. PI 89.1.1.5), L. 88 cm. (C) C2RMF/D. Robcis.

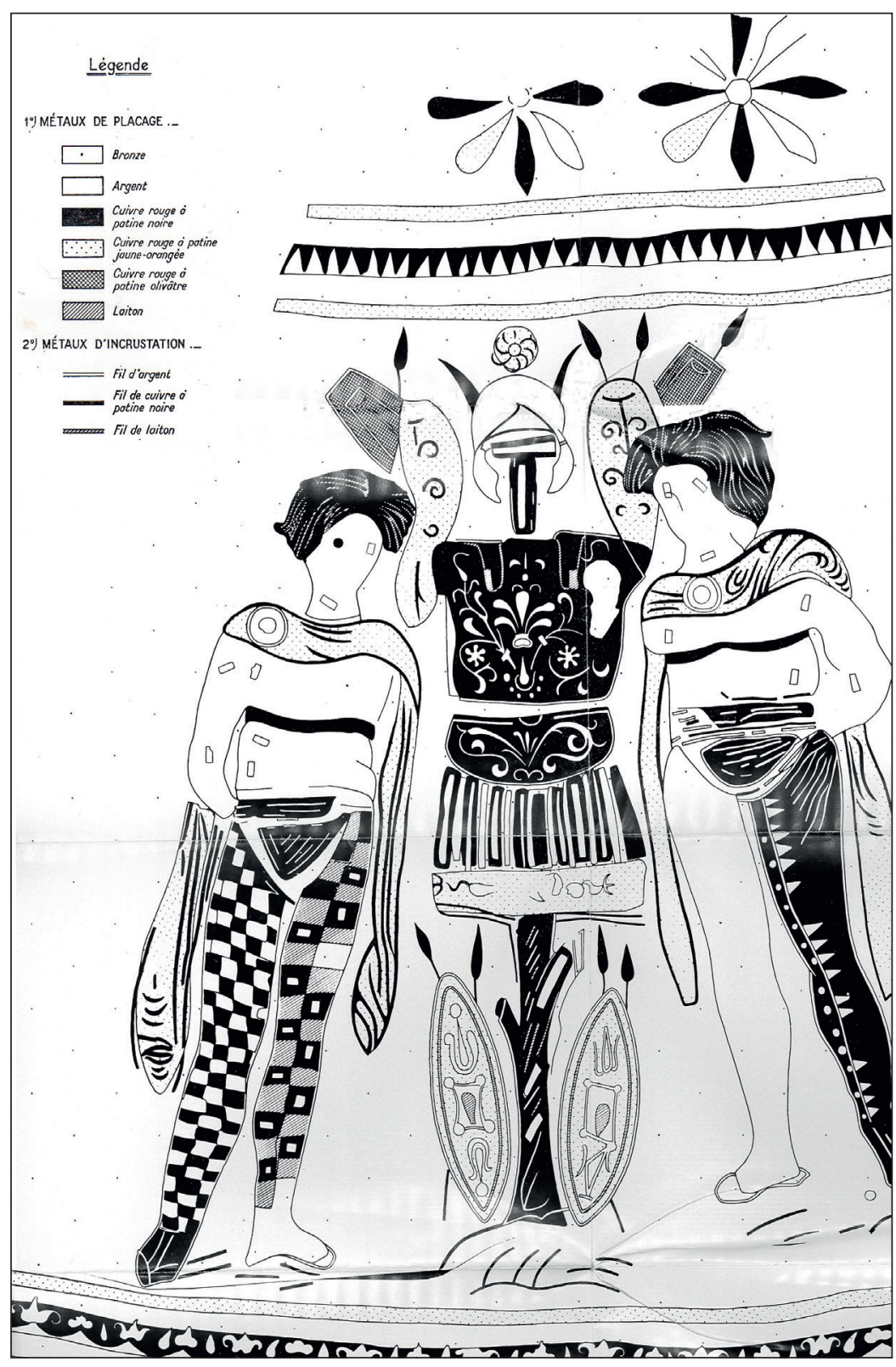

Fig. 4. Partie supérieure du paludamentum de Volubilis, détail du schéma établi par C. Boube-Piccot pour décrire les incrustations. (c) D'après C. Boube-Piccot, 1969. d'incrustations patinées en différentes couleurs. Il est décrit avec précision par les publications de C. Boube-Piccot ${ }^{29}$. La figure 4 montre une partie du schéma établi par cette auteure représentant l'ensemble du décor, assorti des commentaires qu'elle a faits par observation visuelle.

D'après nos propres analyses, le corps de l'objet est une fonte à la cire perdue d'alliage cuivre-étain-plomb contenant de 7 à $15 \%$ en masse d'étain et plus de $10 \%$ de plomb.

C. Boube-Piccot a relevé la présence de cinq alliages d'incrustations : argent, cuivre rouge à patine noire, alliage de cuivre à patine jaune orangée, alliage de cuivre à patine olivâtre, laiton. Les analyses par XRF (fluorescence de rayons $\mathrm{X})$ effectuées par nos soins montrent que l'alliage patiné en noir contient toujours de l'or et de l'argent, de l'arsenic mais pas d'étain (ce qui exclut une influence du substrat sur les analyses). L'analyse par spectroscopie Raman, que nous avons également menée, montre que la patine est de la cuprite $\mathrm{Cu}_{2} \mathrm{O}$. Il s'agit donc bien d'un cuivre noir, tel que l'avait pressenti C. Boube-Piccot par observation visuelle.

Nos analyses montrent que les incrustations blanches sont bien de l'argent, sans que l'on puisse donner plus de précision sur la teneur en cuivre et plomb du fait de l'influence du substrat sur les analyses. Les incrustations rouges à patine orangée et à patine olivâtre sont soit en cuivre non allié, soit en cuivre enrichi notablement de plomb sans que nous ayons pu confirmer les distinctions entre elles ni préciser la nature 
de la patine, sans doute intentionnelle. Les incrustations de laiton sont très lacunaires ; la teneur en zinc de ce laiton, $7,5 \%$, est sous-estimée, du fait de la dézincification due à la corrosion.

La figure 5 donne un exemple de détail. On y voit les chevrons de cuivre noir avec une patine partiellement lacunaire mais très bien conservée par endroits, telle qu'elle a été observée au moment des analyses. De plus, un examen attentif de la surface de l'alliage de base fait soupçonner la présence d'une patine générale pourpre, qui serait logique

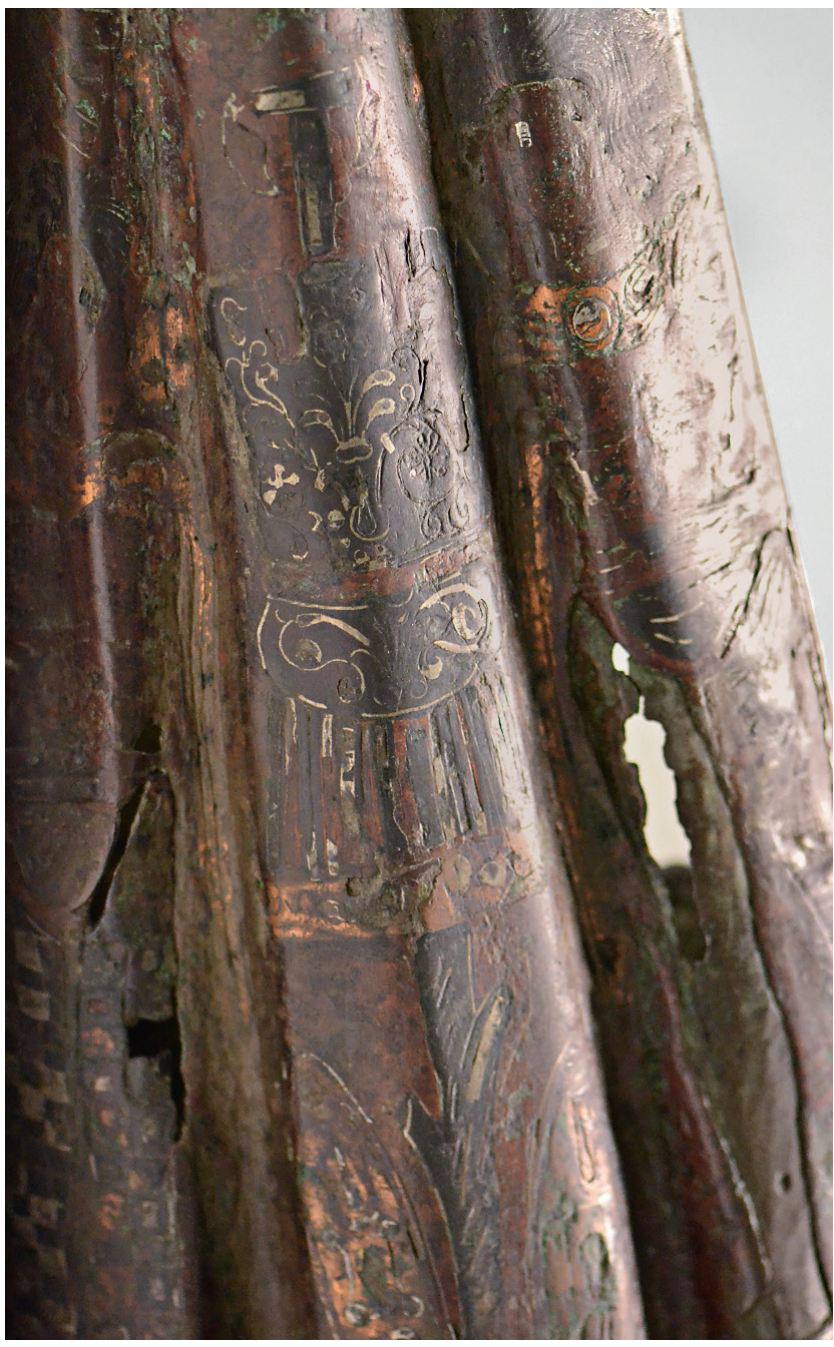

Fig. 5. Détail des incrustations du paludamentum de Volubilis. (c) C2RMF/D. Robcis. pour un paludamentum impérial. Il n'a malheureusement pas été possible de confirmer ce point en raison du mauvais état de la surface.

Il est clair que cet objet extrêmement sophistiqué comportait des incrustations patinées intentionnellement, et en particulier des incrustations de cuivre noir. C'était un témoin important de la fabrication des bronzes noirs dans une période plus tardive que l'encrier de Vaison-la-Romaine et le seul exemple connu jusqu'à présent de l'utilisation de ces techniques sur un bronze monumental. De plus, l'observation des différentes couleurs, de la diversité de compositions des incrustations, probablement patinées intentionnellement même si cela n'a pas toujours été possible de le confirmer par les analyses, semblent indiquer pour cette époque (III ${ }^{\mathrm{e}}-\mathrm{IV}^{\mathrm{e}}$ siècle ap. J.-C.) une pratique de la patine chimique plus fine et davantage aboutie, dans le but d'obtenir une polychromie métallique plus complexe que la simple quadrichromie observée sur l'encrier de Vaison-la-Romaine. Cette évolution technique et artistique avait également été constatée sur un autre objet sensiblement de la même époque étudié au C2RMF : la coupe de Césarée (musée du Louvre, département des AGER, inv Br 4391).

\section{Reconstitutions de patine shakudo}

Dans le but de tenter de comprendre les mécanismes de formation de la patine intentionnelle des bronzes noirs, nous avons engagé un programme de recréation expérimentale. Pour cela, il a fallu, faute de connaître les procédés antiques, utiliser les recettes bien connues d'obtention des patines d'alliages de type shakudo. Ces procédés ont permis de reconstituer la patine noire sur plusieurs alliages de synthèse représentatifs des bronzes antiques ${ }^{30}$. Neuf alliages de compositions proches de celles des objets égyptiens ou romains du Louvre ont été employés : quatre alliages $\mathrm{Cu}-4 \% \mathrm{Au}$; $\mathrm{Cu}-3 \% \mathrm{Au}-1 \% \mathrm{Ag}$; Cu-2 \% Au-3 \% Ag ; Cu-4 \% Ag ; quatre alliages de même composition, mais contenant un ajout de $3 \%$ d'arsenic et enfin un alliage témoin Cu-3\% As. Quatre solutions aqueuses ont été testées, toutes tirées de la littérature japonaise $^{31}$. Le tableau 1 donne la liste des composants de ces solutions, utilisées à température voisine de l'ambiante. Dans ce tableau, l'ingrédient rokusho est une solution contenant de l'acétate de cuivre, du carbonate de calcium et de la soude.

Tableau 1. Composition qualitative des solutions de patinage utilisées

\begin{tabular}{|l|c|c|c|c|c|c|c|}
\hline Dénominations & Rokusho & Acétate de cuivre & Sulfate de cuivre & Alun & Chlorure de sodium & Sel & Vinaigre \\
\hline Niage & oui & & oui & & & & \\
\hline Murakami & & oui & oui & oui & & & \\
\hline Su-tampan & & & oui & & oui & oui & oui \\
\hline Rokusho 4 & & oui & oui & & & oui \\
\hline
\end{tabular}


Toutes les patines, testées par diffraction de rayons $\mathrm{X}$, sont constituées de cuprite $\mathrm{Cu}_{2} \mathrm{O}$, sauf celles dues au bain su-tampan qui contiennent de fortes quantités de chlorure de cuivre. Les analyses par PIXE (émission X induite par particules) à l'accélérateur de particules ${ }^{32}$ indiquent toujours que la cuprite contient une quantité notable de métaux précieux originaires des alliages, et du soufre venant de la solution. Signalons que les patines de bronzes noirs antiques contiennent toujours du soufre, ce qui pourrait être une indication sur la composition des recettes de l'Antiquité. La spectrométrie RBS (rétrodiffusion élastique des particules Rutherford) à l'accélérateur de particules, utilisée pour mesurer l'épaisseur des patines, donne, en cas d'utilisation des trois solutions sans chlorure, 0,2 à 0,3 $\mu$ m d'épaisseur. n'augmente pas l'épaisseur de la couche, effet sans doute associé à un défaut de conductibilité de l'oxyde. Les patines contiennent toujours un certain pourcentage de l'élément précieux or ou/et argent, en général enrichi par rapport au substrat. La présence d'arsenic diminue leur teneur en élément précieux et la concentration en argent est moins enrichie par rapport au substrat que celle en or. L'argent peut même être absent dans la patine si l'alliage contient de l'arsenic.

Ce dernier élément a une influence notable : en plus de sa concentration moins enrichie dans la patine, il favorise la formation rapide d'une couche plus homogène et plus épaisse. Cette influence est plusieurs fois mentionnée dans la littérature, en particulier pour le patinage des shakudo ${ }^{33}$, sans qu'aucune interprétation n'en soit donnée. La patine des alliages contenant de l'arsenic est nettement poreuse, ce qui pourrait expliquer sa plus grande épaisseur.

La couleur des patines varie à la fois avec la composition des alliages et celle des solutions utilisées tout en restant foncée. C'est, pour les conditions expérimentales utilisées ici, le patinage par la solution rokusho 4 qui donne les résultats les plus satisfaisants. L'aspect électrochimique de ces essais est discuté dans la partie suivante.

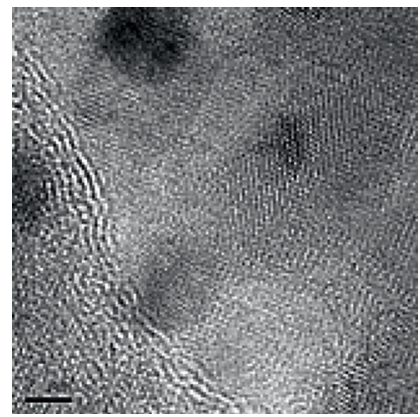

Fig. 6. Microscopie électronique en transmission d'un bronze noir. Nanoparticules noyées dans la cuprite. (C) EMAT, Anvers/E. Grieten.

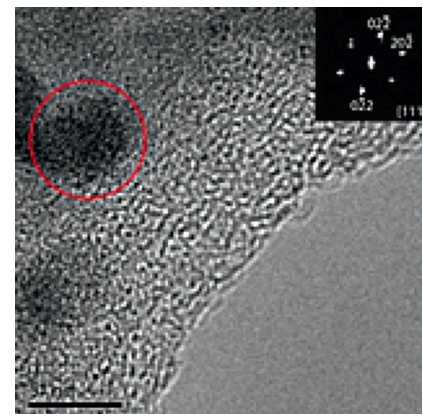

Fig. 7. Microscopie électronique en transmission d'un bronze noir. Nanoparticules noyées dans la cuprite. En insert : diagramme de diffraction électronique d'une particule d'or ou d'argent. (C) EMAT Anvers/E. Grieten.

\section{Microstructure des patines reconstituées ${ }^{34}$}

Pour aider à l'interprétation des causes de la coloration en noir des patines de bronzes contenant des éléments précieux or et/ou argent, un échantillon issu des reconstitutions mentionnées ci-dessus a été choisi pour observer au microscope électronique en transmission (MET) la microstructure de la patine. Il s'agit de l'alliage contenant $3 \%$ d'or et $1 \%$ d'argent, patiné par la solution murakami. Une lame mince transparente aux électrons (environ $500 \mathrm{~nm}$ ) d'une coupe transverse a été obtenue par pulvérisation par les ions d'un canon très focalisé (FIB pour focused ion bombardment).

Les figures 6, 7 et 8 montrent quelques images obtenues par MET, ainsi qu'une analyse de nanoparticules utilisant la méthode EDS (spectrométrie d'émission X par dispersion en énergie). Des spectres de perte d'énergie des électrons (EELS) ont été utilisés pour identifier les phases. Ces figures démontrent sans ambiguité la présence dans la patine de nanoparticules ellipsoïdales ayant une dimension de l'ordre ou inférieure à $5 \mathrm{~nm}$. La microdiffraction des électrons et l'analyse EDS identifient ces particules à l'argent et/ou l'or métallique ; la microdiffraction ne permet pas de différencier $\mathrm{Au}$ et Ag métalliques, mais l'analyse EDS montre bien la présence des deux éléments, simultanément semble-t-il, dans les particules. En dehors des nanoparticules, la couche contient du cuivre, de la cuprite $\mathrm{Cu}_{2} \mathrm{O}$ et de la ténorite $\mathrm{CuO}$. Les proportions de ces différentes phases varient en fonction de la distance au substrat. La présence des nanoparticules explique le fait que la cuprite prenne une couleur noire, comme nous allons l'exposer maintenant.

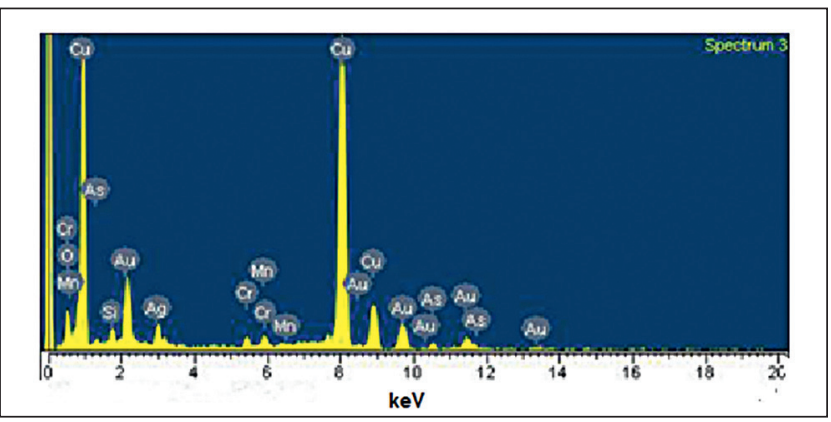

Fig. 8. Spectre d'analyse STEM-EDS sur une nanoparticule métallique de bronze noir. @ Document d'après EMAT Anvers/E. Grieten. 


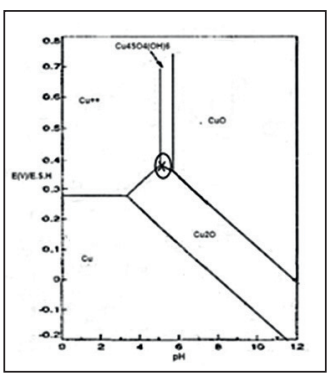

Fig. 9. Diagramme de Pourbaix potentiel-pH du cuivre en présence d'une solution aqueuse contenant du sulfate de cuivre. La croix indique la position du couple potentiel-pH du cuivre pendant le patinage.

(c) D'après Pourbaix, 1966.

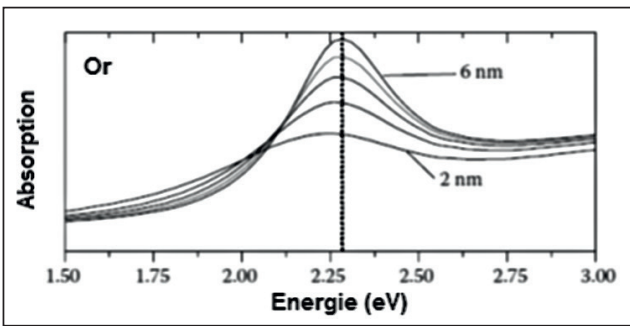

Fig. 10. Diagramme section efficace d'absorptionénergie des photons pour des nanoparticules d'or de différentes dimensions noyées dans une alumine poreuse. (C) D’après Cottancin, 2010.

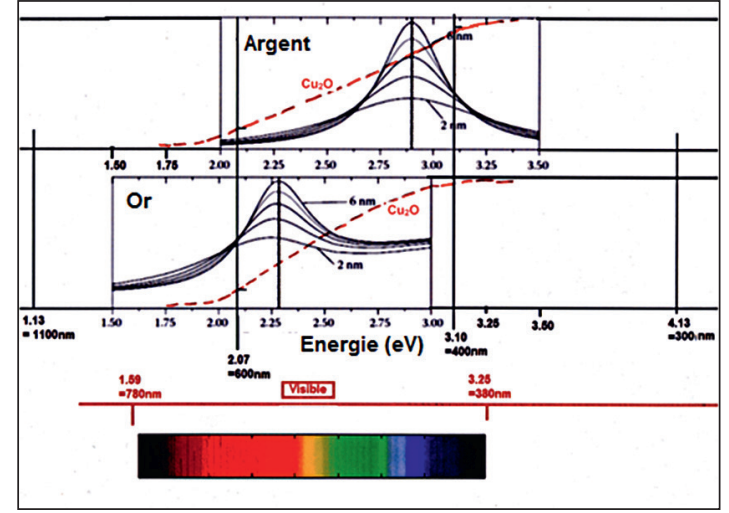

Fig. 11. Reports de la courbe d'absorption lumineuse de la cuprite $\mathrm{Cu}_{2} \mathrm{O}$ sur les diagrammes de section efficace d'absorption de nanoparticules d'argent ou d'or. (C) Document M. Aucouturier d'après Cottancin, 2010.

\section{Discussion sur les mécanismes de formation et la couleur des patines}

\section{Électrochimie du patinage}

Les solutions utilisées dans les recettes japonaises sont des solutions modérément oxydantes. Une étude électrochimique a pu être effectuée il y a quelques années par le laboratoire des Interfaces et des systèmes électrochimiques de l'université Paris-VI (LISE) ${ }^{35}$. Elle a été complétée par des mesures de $\mathrm{pH}$ dans le cadre des reconstitutions décrites au paragraphe précédent.

Si l'on examine le diagramme potentiel-pH du système cuivre-eau avec des ajouts de sulfate (diagramme de Pourbaix) ${ }^{36}$ (fig. 9) et si l'on y place le point correspondant au potentiel électrochimique et au $\mathrm{pH}$ mesurés pendant les expériences de patinage, on constate que ce point se place, grâce à la composition de la solution (ici le bain murakami), mais aussi grâce à la présence de l'or dans l'alliage, élément noble qui augmente le potentiel d'équilibre de l'alliage, à la limite qui sépare les domaines d'existence des ions $\mathrm{Cu}^{++} \mathrm{du}$ composé $\mathrm{Cu}_{4} \mathrm{SO}_{4}(\mathrm{OH})_{6}$ et de la cuprite $\mathrm{Cu}_{2} \mathrm{O}$ (croix sur la figure 9). Cette situation explique en particulier la présence d'un mélange de ténorite $\mathrm{CuO}$ et de cuprite dans la patine. Des mesures chrono-potentiométriques effectuées en condition de réduction confirment bien que la patine est constituée principalement de cuprite et permettent d'estimer l'épaisseur de la couche.

\section{Mécanisme d'apparition de la couleur noire}

Le comportement optique de nanoparticules métalliques dans une matrice diélectrique a fait l'objet d'études physiques nombreuses et très complètes ${ }^{37}$. Du fait de la faible taille de ces particules conductrices, il apparaît en surface de celles-ci, quand elles sont excitées par un rayonnement électromagnétique (la lumière), un effet de résonance en surface des électrons de conduction (surface plasmon resonance, SPR). Ceci a pour conséquence une forte absorption de la lumière visible dans une gamme limitée de longueurs d'onde. L'application de la théorie de $\mathrm{Mie}^{38}$ à un solide diélectrique comportant des nanoparticules métalliques soumises à la SPR donne des pics d'absorption de la lumière dont l'énergie (la longueur d'onde) dépend de la nature et de la composition des particules, et dont la largeur en énergie est liée à la taille de ces dernières. La figure 10 montre le cas de nanoparticules d'or noyées dans une alumine poreuse ${ }^{39}$. On y remarque que le pic d'absorption se situe à une énergie de 2,30 eV environ et que, pour une taille de nanoparticules d'or de $5 \mathrm{~nm}$, la largeur du pic avoisine $0,4 \mathrm{eV}$.

On peut alors superposer les pics d'absorption SPR des nanoparticules d'argent ou d'or présentes dans une patine de bronze noir au spectre d'absorption de la cuprite $\mathrm{Cu}_{2} \mathrm{O}$ obtenu dans la littérature ${ }^{40}$ (fig. 11). On y constate alors que la partie de basse énergie du spectre d'absorption de la cuprite, qui montre une absorption très faible - manifestation de sa couleur rouge -, est fortement occultée par les pics de Mie liés à la présence des nanoparticules, et ceci d'autant mieux que les particules sont petites. La couleur rouge a donc tendance à disparaître avec, comme conséquence, le noircissement de la patine, qui se trouve ainsi justifié.

Il reste à expliquer la raison de la présence de ces nanoparticules de métaux précieux au sein de la patine. Il faut pour cela faire encore appel à l'électrochimie : compte tenu du potentiel d'électrode pendant la formation de la patine, seul le cuivre s'oxyde préférentiellement, laissant sur place les atomes d'or et d'argent métalliques qui s'agglomèrent en nanoparticules, car la température basse ne leur permet pas de diffuser sur de grandes distances. 


\section{Conclusion}

L'existence de bronzes patinés en noir par des procédés chimiques, attestée depuis la haute Antiquité, a fait l'objet d'un grand nombre de publications, fondées sur la description d'objets de musées ou d'incrustations sur ces objets. Mais il y a peu de travaux sur les phénomènes électrochimiques intervenant pendant l'opération de patinage ou sur les mécanismes optiques de la transformation colorée du rouge au noir de la couche de cuprite $\mathrm{Cu}_{2} \mathrm{O}$ ainsi formée.

Le présent article apporte quelques éléments de réponse à ces deux questions. L'ajout de métaux précieux or et/ou argent dans l'alliage ainsi que la composition du bain de patinage créent des conditions électrochimiques propices à la formation préférentielle d'une couche de cuprite $\mathrm{Cu}_{2} \mathrm{O}$ en surface. Nous avons par ailleurs confirmé que la perte de la couleur naturelle rouge de cette cuprite au profit d'une teinte noire ou bleu-noir s'explique assez bien par un effet dit surface plasmon resonance $(S P R)$ à la surface des nanoparticules d'or et/ou d'argent présentes dans la couche de cuprite. L'étude faite par microscopie électronique en transmission a permis de préciser la taille de ces nanoparticules, inférieure ou égale à $5 \mathrm{~nm}$. Grâce à la théorie de Mie, on sait qu'une taille aussi petite favorise l'étalement en énergie des pics d'absorption lumineuse, donc l'occultation de la couleur rouge de la cuprite.

Il reste quelques questions non encore résolues. Ainsi, le rôle incontestablement bénéfique de l'arsenic en addition dans les alliages reste incompris. Une autre propriété non expliquée est l'exceptionnelle stabilité dans le temps de la patine noire. Certains chercheurs font même état de sa capacité de régénération spontanée en cas de destruction accidentelle localisée (rayure) ${ }^{41}$ ! Enfin, faute de documents antiques fiables, nos études n'ont pas beaucoup fait progresser la connaissance des procédés de patinage et le degré de parenté de la ou les recettes antiques avec les recettes japonaises connues. Une collaboration étroite entre philologues, historiens des techniques et spécialistes des alchimistes grecs pourrait peut-être apporter des éléments de réponse.

\section{Remerciements}

Outre les stagiaires mentionnées en notes, nous tenons à remercier Élisabeth Delange, conservateur en chef au département des Antiquités égyptiennes du musée du Louvre, et Sophie Descamps-Lequime, conservateur général au département des Antiquités grecques, étrusques et romaines de ce même musée, qui ont mis à notre disposition les précieux objets du Louvre placés sous leur responsabilité, et ont amplement participé aux études scientifiques de ces objets, en les replaçant dans leur contexte historique et culturel.

Nous tenons aussi à exprimer notre reconnaissance à l'équipe de l'accélérateur AGLAE qui nous a totalement secondés dans les analyses chimiques, en les personnes du regretté Joseph Salomon, à qui cet article est dédié, et de ses collègues Laurent Pichon et Brice Moignard, ainsi qu'à Michel Dubus pour son aide en diffraction des rayons X.

\section{Notes}

1. La différence entre patine «volontaire " et patine « naturelle " est bien discutée dans l'article de Techné (Aucouturier, 2003 - voir note 2). Pour répondre à une objection de lecteur anglophone, nous préférons désormais l'expression "patine intentionnelle », sachant que certaines patines intentionnelles sont obtenues par voie naturelle (toit de l'opéra Garnier ou de la basilique de Saint-Denis).

2. Aucouturier, 2003 ; Aucouturier,

2010 ; Craddock, 1993, p. 101-127.

3. Giumlia-Mair, 1996.

4. Descamps-Lequime, 2005.

5. Craddock, 1993, p. 101-127.

6. Murakami, 1993, voir note 2, p. $85-94$

7. Stone, 2010 ; Stone, 2011, p. 178-182 ; Hugues, 1991

8. Viano et Martelli, 2016 ; et Conférences prononcées au workshop «Pour une approche interdisciplinaire de l'alchimie grecque : autour $d u$ verre, du cinabre et du bronze noir ", en collaboration avec le C2RMF au Louvre et à la Maison de la Recherche, le 18-19 novembre 2009.

9. Berthelot, 1885 ; Berthelot, 1888-1889.
10. Halleux, 1981.

11. Hunter, 2002.

12. München Staatliches Museum, Ägyptisher Kunst, inv. AS 6080 ; GiumliaMair, 1996, voir note 3 .

13. Mathis, 2009

14. Craddock, 1993, p. 101-127.

15. Cooney, 1966 ; Giumlia-Mair, 1997.

16. Mathis, 2009

17. Musée national archéologique d'Athènes (inv. NAMA 394) ;

Demakopoulou, 1995.

18. Voir Dubel, 2006, p. 161-181 et les références ci-incluses ; $\mathrm{S}$. Descamps-

Lequime, 2006, ibid., p. 79-92.

19. Dubel, 2006, ibid.

20. Pline l'Ancien, NH 34.1 et NH 9.65.

21. Descamps-Lequime, 2015 ;

Descamps-Lequime, 2005.

22. Descamps-Lequime, 2005.

23. Mathis, 2005a.

24. Mathis, 2009, voir note 13 ; voir note

2.

25. Robcis, 2015

26. Mathis, 2005b.

27. Exp. Marseille, 2014 [Marseille,

MUCEM, 12 mars-25 août 2014] p. 114-117 ;

Boube-Piccot, 2014, ibid., p. 35.

28. Nous remercions Clarisse Lebas, commissaire, qui a accepté, grâce à l'aimable intercession de Sophie Descamps-Lequime, conservateur général au musée du Louvre, de mettre cet objet à notre disposition pendant le temps des analyses faites sur place au MUCEM.

29. Boube-Piccot, 1966 , p. 189-278 ; Boube-Piccot, 1969, p. 88-103 ; BoubePiccot, 2014.

30. Nous sommes reconnaissants à Sophie Tirat qui a effectué ces essais dans le cadre d'un stage à l'université de Liège pour son Master de chimie de l'université Pierreet-Marie-Curie de Paris.

31. Voir note 6 .

32. Calligaro, 2004

33. Craddock, 1993, voir note 2, p. 102 ; Giumlia-Mair, 2001.

34. Nous sommes très reconnaissants à Eva Grieten pour les observations effectuées au laboratoire EMAT de l'université d'Anvers, sous la direction du professeur Dominique Schryvers.

35. Nous sommes reconnaissants à Michel Keddam et Hisasi Takenouti du laboratoire LISE (Paris-VI), avec qui cette étude a été menée en collaboration dans le cadre du stage de Lucile Klein. Les résultats principaux en sont publiés dans Aucouturier, 2003 (voir note 2).

36. Pourbaix, 1966, p. 357. 
37. Par exemple : Cottancin, 2010,

p. 24-1 à 24-7.

38. Mie, 1908.

39. Voir note 37 , p. $24-7$

40. Zouaghi, 1968.

41. A. Giumlia-Mair, communication personnelle, 2000.

\section{Bibliographie}

Aucouturier M., Keddam M., Robbiola L., Takenouti H., 2003, « Les patines des alliages de cuivre : processus naturel ou œuvre de l'homme? ", Techné, 18, p. 86-94.

Aucouturier M., Mathis F., Robcis D., Castaing J., Salomon J., Pichon L., Delange E., Descamps-Lequime S. 2010, "Intentional patina of metal archaeological artefacts: nondestructive investigation of Egyptian and Roman museum treasures", Corrosion Engineering, Science and Technology, $45 \mathrm{~N}^{\circ}$ 5, p. 314-321.

Berthelot M., 1885, Les origines de l'alchimie, Georges Steinhel, Paris.

Berthelot M., Ruelle C. E., 1888-1889, Collection des anciens alchimistes grecs, réédition Nabu Press, Paris 2013.

Boube-Piccot C., 1966, « Trophée damasquiné sur une statue impériale de Volubilis ", Bulletin d'archéologie marocaine 6, Rabat, p. 189-278.

Boube-Piccot C., 1969, Les bronzes antiques du Maroc, partie I La statuaire, musée des Antiquités, Rabat, p. 88-103.

Boube-Piccot, 2014, « Les bronzes antiques du Maroc ", dans Exp. Marseille, 2014, Splendeurs de Volubilis. Bronzes antiques du Maroc et de Méditerranée, p. 32-37.

Calligaro T., Dran J.-C., Salomon J., 2004, "Ion beam analyses", dans Janssens K., Van Grieken R., Non destructive microanalysis of cultural heritage materials, Comprehensive Analytical Chemistry, Elsevier, Amsterdam, Vol. XLII, 2004, p. 227-276.

Cooney J. D., 1966, "On the meaning of Hsmn-Km”, Zeitschrift für Ägyptische Sprache und Altertumskunde, 93, p. 43-47.

Cottancin E., Broyer M., Lermé J., Pellarin M., 2010, "Optical properties of metal clusters and nanoparticles", dans Sattler K. D. (dir.), Handbook of Nanophysics: Nanoelectronics and Nanophotonics, p. 24-1 à 24-7, et publications associées.

Craddock P., Giumlia-Mair A., 1993, "Hsmn$\mathrm{Km}$, Corinthian bronze, Shakudo, black patinated bronze in the ancient world", dans Craddock P., La Niece S. (dir.), Metal plating and patination, Butterworth Heinemann, Oxford, p. 101-127.

Demakopoulou K., Mangou E., Jones R. E., Photos-Jones E., 1995, "Mycenaean and black inlaid metalware in the National Archaeological Museum, Athens. A technical examination", The Annual of the British School of Athens, 90, p. 137-153.

Descamps-Lequime S., 2005, « L'encrier de Vaison-la-Romaine et la patine volontaire des bronzes antiques", Monuments et Mémoires de la fondation Eugène-Piot, 84, p. 5-30.
Descamps-Lequime S., 2006, « La polychromie des bronzes grecs et romains » dans Rouveret A., Dubel S. e Naas V. (dir.), Couleurs et matières dans l'Antiquité, éditions Presses de l'E.N.S., Paris, p. 79-92.

Descamps-Lequime S., 2015, "The color of bronze. Polychromy and the aesthetics of bronze surface", dans Exp. FlorenceLos Angeles-Washington, 2015, Power and Pathos. Bronze Sculpture of the Hellenistic World, p. 151-165.

Dubel S., 2006, «Quand la matière est couleur. Du bouclier d'Achille aux "tableaux de bronze de Taxila” » dans Rouveret A., Dubel S. et Naas V. (dir.), Couleurs et matières dans l'Antiquité, éditions Presses de l'E.N.S., Paris, p. 161-181.

Exp. Florence-Los Angeles-Washington, 2015 : Power and Pathos. Bronze Sculpture of the Hellenistic World [Exposition, Florence, Palazzo Strozzi, 14 March21 June 2015 ; Los Angeles, J. Paul Getty Museum, 28 July-1 November 2015 ; Washington D.C., National Gallery of Art, 6 December 2015-20 March 2016, Daehner J. M., Lapatin K. (eds.)], Giunti Editore, Fondazione Palazzo Strozzi, J. Paul Getty Trust.

Exp. Marseille, 2014, Splendeurs de Volubilis. Bronzes antiques du Maroc et de Méditerranée [Marseille, MUCEM, 12 mars-25 août 2014, Deledalle M. M. (dir.)], notice par Abdelaziz El Khyari p. 114-117, musée archéologique de Rabat, inv. PI 89.1.15.

Giumlia-Mair A., 1996, «Das Krokodil und Amenemhat III aus el-Fayum ", Antike welt 4, p. 313-321.

Giumlia-Mair A., Quirke S., 1997, "Black copper in Bronze Age Egypt", Revue d'Égyptologie 48, p. 95-108.

Giumlia-Mair A., Lehr M., 2001, "Experimental reproduction of artificially patinated alloys, identified in ancient Egyptian, palestinian, mycenaean and roman objects", dans Archeologie sperimentali-Metodologie ed esperienze fra verifica, riproduzione, communicazione e simulazione, (Cormano Terme - Fiavè, Trento, Italie, 2001).

Halleux R., 1981, Les alchimistes grecs. 1. Papyrus de Leyde. Papyrus de Stockholm. Fragments de recettes, Les Belles Lettres, Paris.

Hughes R., Rowe M., 1991, The colouring, bronzing and patination of metals, WatsonGuptill Publications, New York.

Hunter E. C. D., 2002, "Beautiful black bronzes: Zosimo's treatises in Cam. Mm.6.29" dans Giumlia-Mair A. (ed.), Bronzi Antichi: Produzione e tecnologia, actes $d u X V^{e}$ congrès International sur les bronzes antiques, (Aquiliea 22-26 mai 2001), Università di Udine, 2002.

Mathis F., 2005a, Croissance et propriétés des couches d'oxydation et des patines à la surface d'alliages cuivreux d'intérêt archéologique ou artistique, Thèse Université Paris Sud, disponible sur https://tel.archives-ouvertes.fr/tel00011255 . Consulté en novembre 2016.

Mathis F., Descamps S., Robcis M., Aucouturier M., 2005b, “Original surface treatment of copper alloy in ancient Roman Empire: chemica patination on a Roman strigil", Surface Engineering, 21, p. 346-351.

Mathis F., Delange E., Robcis D., Aucouturier M., 2009, "HMTY-KM (black copper) and the Egyptian bronzes' collection of the musée du Louvre", Journal of Cultural Heritage, 10, p. $63-72$.

Mie G., 1908, « Beiträge zur Optik trüber Medien speziell kolloidaler Goldlosungen (contributions to the optics of diffuse media, especially colloid metal solutions) ", Ann Phys, 25, p. $377-445$.

Murakami R., 1993, "Japanese traditionnal alloys”, dans Craddock P., La Niece S. (dir.) Metal plating and patination, Butterworth Heinemann, Oxford, p. $85-94$.

Photos E., Jones R. E., Papadopoulos Th., 1994, "The black inlay decoration on a Mycenian bronze dagger", Archaeometry, 36 , issue 2, p. 267-275.

Pline l'Ancien, Histoire Naturelle, Traduction J. M. Croisille, NH 34.1 et NH 9.65, Les Belles Lettres, Paris, 1985.

Pourbaix M., 1966, Atlas d'équilibres électrochimiques, Gauthier-Villars, Paris, p. 357.

Robcis D., Aucouturier M., Descamps S., Marsac J., Lambert E., Langlois J., Lemasson Q., Moignard B., Pacheco C., Pichon, L., 2015, « La prise en compte des surfaces et des patines antiques dans la restauration des métaux archéologiques : étude de la plaque à l'Aurige » dans S. Clerbois (dir.), La conservation-restauration des métaux archéologiques : des premiers soins à la conservation durable, Les dossiers de l'IPW 15, Institut du patrimoine wallon, Bruxelles, p. 67-75.

Stone R. E., 2010, "Organic patinas on small bronze of the Italian Renaissance", Metropolitan Museum Journal, 45, p. 107-124.

Stone R. E., 2011, “Understanding Antico’s patina”, dans E. Luciano (dir.), Antico: The Golden Age of Renaissance Bronzes, catalogue d'exp., The National Gallery of Art, Washington, 6 November 20118 April, 2012, p. 178-182.

Viano C., Martelli M. (dir.), 2016, Les alchimistes grecs. Textes, doctrines, comparaisons. Actes du colloque (Venise 5-7 décembre 2007), université de Venise Ca' Foscari et Centre Léon Robin, UMR 8061, CNRS, Paris.

Zouaghi M., Fortin E., Zielinger, 1968, «Quelques propriétés optiques et de transport des couches minces de cuprite ", Journal de Physique, 29, p. C2-75-C2-82. 